\title{
A new green approach for the reduction of graphene oxide nanosheets using caffeine
}

\author{
THU HA THI VU ${ }^{1, *}$, THANH THUY THI TRAN ${ }^{1}$, HONG NGAN THI LE ${ }^{1}$, \\ PHUONG HOA THI NGUYEN ${ }^{1}$, NGOC QUYNH BUI ${ }^{2}$ and NADINE ESSAYEM ${ }^{2}$ \\ ${ }^{1}$ Key Laboratory for Petrochemical and Refinery Technologies, Hanoi, Vietnam \\ ${ }^{2}$ Institute of Researches on Catalysis and Environment in Lyon, CNRS, Lyon 1, 69626 Villeurbanne, France
}

MS received 9 September 2014; revised 14 November 2014

\begin{abstract}
A simple and green chemistry approach for the preparation of reduced graphene oxide nanosheets was successfully demonstrated through the reduction of graphene oxide (GO) using caffeine as the reductant. Without using toxic and harmful chemicals, this method is environmentally friendly and suitable for the large-scale production of graphene. The samples of GO, before and after reduction with caffeine have been characterized by $\mathrm{X}$-ray diffraction, Raman, Fourier transform infrared, X-ray photoelectron spectroscopy, thermogravimetric analysis and transmission electron microscopy.
\end{abstract}

Keywords. Graphene; caffeine; graphene oxide; reduced graphene oxide; green chemistry.

\section{Introduction}

In recent years, graphene has become a 'miracle material' owing to its unique physical and chemical properties. ${ }^{1,2}$ It has a great potential in various applications such as conversion or charge storage, ${ }^{3,4}$ drug delivery, ${ }^{5,6}$ sensors $^{7}$ and composite materials. ${ }^{8}$ Graphene can be synthesized by chemical vapour deposition, ${ }^{9}$ ultrasonication, ${ }^{10}$ electrochemical method, ${ }^{11}$ chemical methods ${ }^{12}$ and so on. Among these methods, the chemical method based on reduction of graphene oxide (GO) has been developed for the large-scale production of graphene. Hydrazine is the most widely used reducing agent for GO. ${ }^{13}$ However, because of its high toxicity, the research for alternative green reductant is strongly demanding. In recent times, many attempts have been made to develop green reducing agents such as glucose, ${ }^{14}$ ascorbic acid, ${ }^{15}$ melatonin, ${ }^{16}$ green tea, ${ }^{17}$ microbial, ${ }^{18}$ formamidine sulfurnic acid, ${ }^{19}$ drained water from soaked mung beans ${ }^{20}$ and L-glutathione. ${ }^{21}$

The aim of this work is to prove that caffeine can also be used for scalable preparation of high-quality reduced GO (rGO) using natural graphite as raw material. Caffeine is an alkaloid that is found in varying quantities in the seeds, leaves and fruits of some plants. It is most commonly consumed by humans in infusions extracted from the seed of the coffee plant and the leaves of tea bush as well as from various foods and drinks containing products derived from the kola nut. Caffeine could be used as green reductant as it is nontoxic, biocompatible and commercially available at industrial level. Therefore, the production of nanosized graphene

\footnotetext{
*Author for correspondence (ptntd2004@yahoo.fr)
}

sheets using caffeine is suitable for broad biomedical applications including drug delivery, bioimaging and biosensing. In the present work, the transformation of GO to rGO was characterized by various surface characterization techniques including X-ray diffraction (XRD), Raman, Fourier transform infrared (FT-IR), X-ray photoelectron spectroscopy (XPS), thermogravimetric analysis (TGA) and transmission electron microscopy (TEM).

\section{Experimental}

\subsection{Materials}

Graphite powder, potassium permanganate, 98\% sulphuric acid, $30 \%$ hydrogen peroxide, $35 \%$ hydrochloric acid, ethanol and caffeine were all purchased from Sigma-Aldrich and used directly without further purification. All aqueous solutions were prepared with Milli-Q water (Purelab Option-Q).

\subsection{Preparation of $G O$}

GO was prepared by oxidizing the graphite powder in a mixture of concentrated sulphuric acid and $\mathrm{KMnO}_{4}$ according to the modified Hummers method. ${ }^{22}$ Briefly, $2 \mathrm{~g}$ of graphite powder was stirred in $35 \mathrm{ml}$ of $98 \% \mathrm{H}_{2} \mathrm{SO}_{4}$ on a magnetic stirrer for $2 \mathrm{~h}$. Then $6 \mathrm{~g}$ of $\mathrm{KMnO}_{4}$ was gradually added into the above solution by maintaining the temperature below $20^{\circ} \mathrm{C}$. The mixture was then stirred at $35^{\circ} \mathrm{C}$ for $4 \mathrm{~h}$ in a water bath. The resulting solution was diluted by adding $90 \mathrm{ml}$ of water under vigorous stirring for $1 \mathrm{~h}$. Then a dark brown suspension was obtained. The suspension was further treated by adding $30 \% \mathrm{H}_{2} \mathrm{O}_{2}$ solution drop wise until the colour of the 
solution became bright yellow. The resulting GO suspension was washed by repeated centrifugation, first with $5 \%$ aqueous $\mathrm{HCl}$ solution to remove excess of manganese salt followed by water until the $\mathrm{pH}$ of the solution became neutral. The purified GO was finally dispersed in water $\left(0.5 \mathrm{mg} \mathrm{ml}^{-1}\right)$ ultrasonically in an ultrasonic bath. Thus a stable dispersion of GO was obtained.

\subsection{Reduction of $G O$}

A mixture $(20 \mathrm{ml})$ of $\mathrm{GO}$ at concentration of $0.1 \mathrm{mg} \mathrm{ml}^{-1}$ and caffeine at concentration of $3 \mathrm{mg} \mathrm{m}^{-1}$ were maintained at $80^{\circ} \mathrm{C}$ for $12 \mathrm{~h}$. Dispersions were sonicated for $30 \mathrm{~min}$ prior to experiments by a ultrasonic bath and continuously stirred throughout the reaction. A homogeneous reduced GO (rGO) dispersion was obtained without aggregation. Solid rGO was obtained by centrifugation at $14,500 \mathrm{rpm}$ for $30 \mathrm{~min}$, and then washed many times with hot distilled water and ethanol to remove caffeine and its derivatives after reduction. For each washing, the solution was again centrifuged $14,500 \mathrm{rpm}$ for 30 min and the supernatant was discarded. The final product was dried at $45^{\circ} \mathrm{C}$ overnight in a vacuum oven.

\subsection{Characterization of $G O$ and $r G O$}

XRD analyses were performed on D8 Advance-Bruker diffractometer at $40 \mathrm{kV}$ and $15 \mathrm{~mA}$. Raman spectra were recorded using a LabRam HR (Horiba JobinYvon) spectrometer. FT-IR spectra were carried out on Nicolet 6700 Thermo Fisher. XPS analyses were performed in a KRATOS Axis Ultra DLD spectrometer with an $\mathrm{Al} \mathrm{K} \alpha$ monochromatic $(h v=1486.6 \mathrm{eV}) \mathrm{X}$ source. TGA plots of the samples were measured using a Diamond TG/DTA analyzer (Perkin Elmer Instruments). The morphological characteristics of GO and rGO sheets were obtained using a TEM (JEOL-JEM-1100).

\section{Results and discussion}

Figure 1 shows XRD patterns of raw graphite, GO and rGO. The XRD pattern of graphite shows an intensive ${ }^{23}$ peak at $2 \theta=26.6^{\circ}(d$-spacing $=0.335 \mathrm{~nm})$ corresponding to 002 reflection of the graphitic structure. Upon oxidation of graphite to GO, the 002 reflection peak shifts to a lower angle at $2 \theta=11.1^{\circ}(d$-spacing $=0.794 \mathrm{~nm})$. The increase in $d$-spacing is due to the intercalation of water molecules and the formation of oxygen moieties in the interlayer spacing of graphite. The complete transformation from GO to rGO is clearly illustrated by the disappearance of the 002 reflection line with no new diffraction peaks in the XRD pattern is obtained. The XRD pattern of rGO is that of an amorphous material.

Figure 2 illustrates the Raman spectra of GO before and after reduction with caffeine. The $\mathrm{D}$ and $\mathrm{G}$ bands for $\mathrm{GO}$ and rGO are observed at 1349 and $1594 \mathrm{~cm}^{-1}$, respectively. The $\mathrm{D}$ band indicates the structural defects and disorder, whereas the $\mathrm{G}$ band represents the graphitic component in the structure. Prominently, the intensity ratio of the D and G

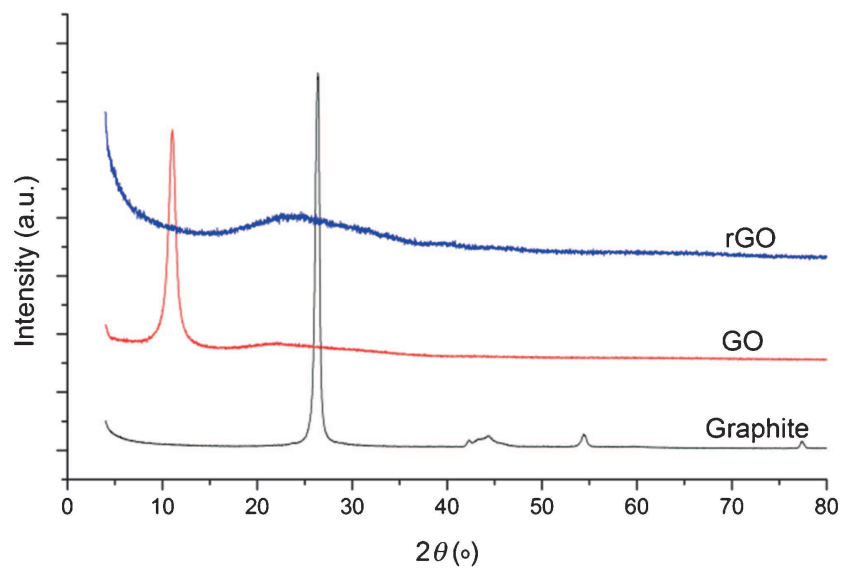

Figure 1. XRD spectra of graphite, GO and rGO.

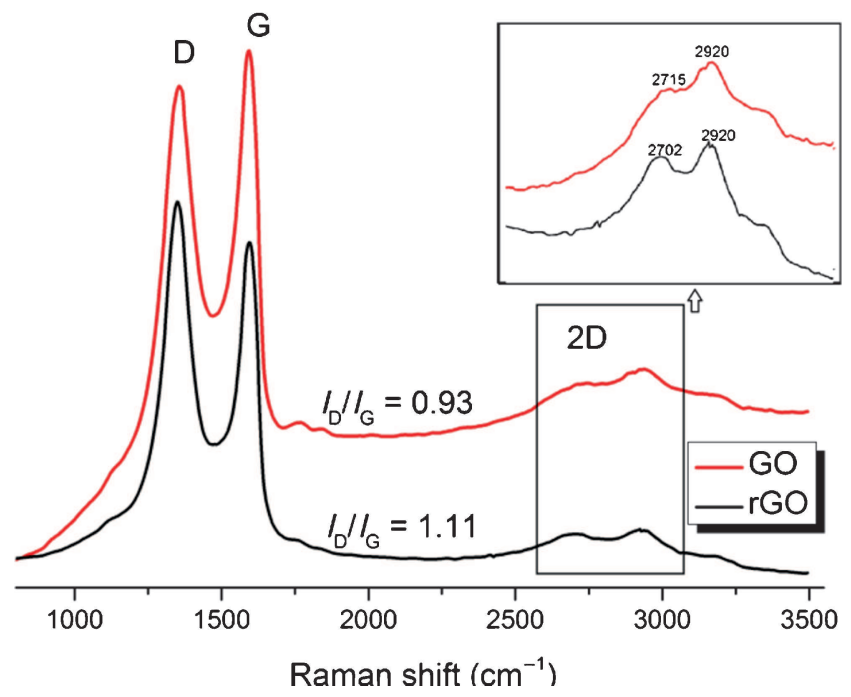

Figure 2. Raman spectra of GO and rGO.

band $\left(I_{\mathrm{D}} / I_{\mathrm{G}}\right)$ of $\mathrm{GO}$ after reduction with caffeine increases from $0.93(\mathrm{GO})$ to 1.11 , indicating an increase in disordered domains during the reduction of GO. ${ }^{23}$ This change of $I_{\mathrm{D}} / I_{\mathrm{G}}$ value is in good agreement with observations reported for the reduction of GO with hydrazine ${ }^{13}$ and other systems. ${ }^{23,24}$ In addition, it was noted that the 2D band of rGO shifted to $2702 \mathrm{~cm}^{-1}$ in comparison with that of GO observed at $\sim 2715$ $\mathrm{cm}^{-1}$. It was previously proposed that the reduction reaction favour the transformation of multilayer GO to further exfoliation into a fewer-layer or single-layer graphene. ${ }^{25}$ The band at $\sim 2920 \mathrm{~cm}^{-1}$ was ascribed to the defect-related band.

The FT-IR spectra of caffeine, GO and rGO are exhibited in figure 3 . In the spectrum of caffeine, there are several characteristic absorptions: the bands at 3093 and $2953 \mathrm{~cm}^{-1}$ corresponds to $\mathrm{N}-\mathrm{H}$ and $\mathrm{C}-\mathrm{H}$ stretching of heterocyclic compounds, while the strong bands at 1699 and $1659 \mathrm{~cm}^{-1}$ are attributed to the two carbonyl groups in the molecule and an additional absorption band at $1539 \mathrm{~cm}^{-1}$ of $\mathrm{C}=\mathrm{N} .^{26}$ For GO, the hydrogen bonding $\mathrm{O}-\mathrm{H}$ stretching vibrations at $3000-3500 \mathrm{~cm}^{-1}$ (broad), and the $\mathrm{O}-\mathrm{H}$ bending near 1400 
$\mathrm{cm}^{-1}$ (narrow), the $\mathrm{C}=\mathrm{O}$ (carboxyl groups) stretching vibrations at $1724 \mathrm{~cm}^{-1}$, the $\mathrm{C}=\mathrm{C}$ skeletal stretching vibrations at $1640 \mathrm{~cm}^{-1}$ which may overlap by the $\delta_{\mathrm{O}-\mathrm{H}}$ of molecular water. At lower wave numbers range, $1300-100 \mathrm{~cm}^{-1}$, are rather characteristic of the $\mathrm{C}-\mathrm{O}$ stretching vibrations (epoxy and alkoxy). In general, the FT-IR spectrum of rGO is quite similar to that of GO. However, the intensity of all the peaks correlated to the oxygen functional groups of $\mathrm{rGO}$ decreases dramatically. Otherwise, the typical absorption bands of caffeine as well as any new absorption bands of caffeine derivatives after reduction cannot be also observed in the FT-IR spectrum of rGO. This fact indicates that caffeine and its derivatives are completely removed from $\mathrm{rGO}$ by washing. These observations, therefore, could prove that most of oxygen-containing groups onto the GO nanosheets are removed in the reduction process using caffeine as the reducing agent.

In order to further illustrate the reduction extent of GO with caffeine, the $\mathrm{C}$ 1s XPS spectra of GO and rGO are used to prove the removal of the oxygen-containing groups (figure 4). For $\mathrm{GO}$ sample, the $\mathrm{C} 1 \mathrm{~s}$ bands can be deconvoluted in three bands attributed to $\mathrm{C}-\mathrm{C}, \mathrm{C}=\mathrm{O}$ and $\mathrm{C}(\mathrm{O}) \mathrm{OH}$ bonds at 284.7, 286.6 and $288.2 \mathrm{eV}$, respectively. ${ }^{27}$ The $\mathrm{C} 1 \mathrm{~s}$ XPS spectrum of rGO shows a significant intensity decrease of $\mathrm{C}=\mathrm{O}$ and $\mathrm{C}(\mathrm{O}) \mathrm{OH}$ bands and an appearance of $\mathrm{C}-\mathrm{O}$ band at $285.5 \mathrm{eV}$ in comparison to that of $\mathrm{GO}$, indicating effective deoxygenation of $\mathrm{GO}$ after the chemical reduction. For rGO sample, the peaks of $\mathrm{C}-\mathrm{C}, \mathrm{C}=\mathrm{O}$ and $\mathrm{C}(\mathrm{O}) \mathrm{OH}$ bands locate at 284.5, 286.8 and $288.7 \mathrm{eV}$, respectively. Thus, there are the shifts in binding energy values of these bands of rGO. These shifts may due to the change in chemical environment of carbon atoms after reduction. ${ }^{20}$ In addition, after reduction process, the surface $\mathrm{C} / \mathrm{O}$ atomic ratio increases from 1.96 (GO) to 6.5 (rGO). These results show that the removal of oxygencontaining groups of $\mathrm{GO}$ using caffeine as reduction reagent was relatively successful.

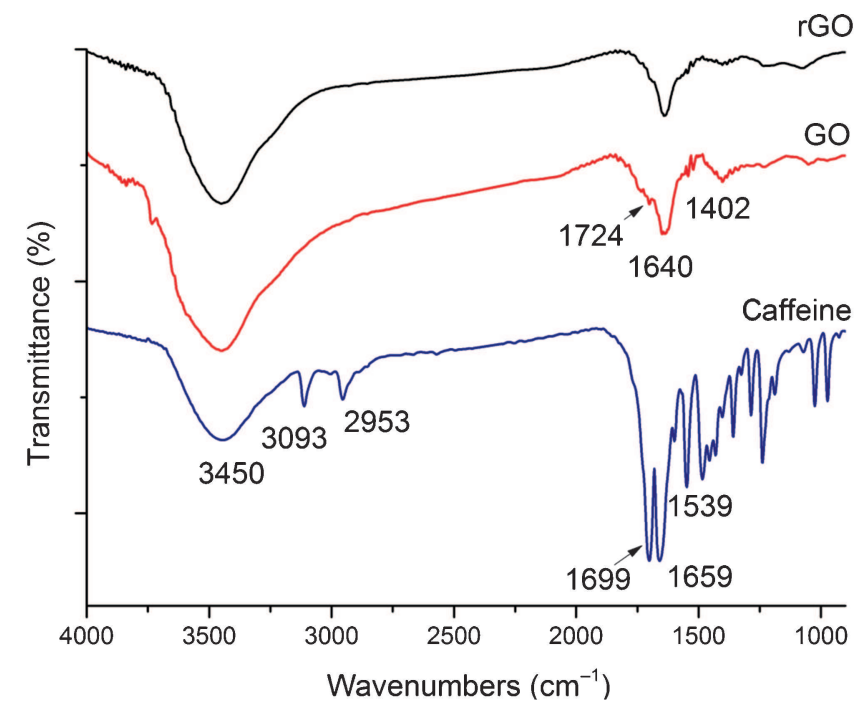

Figure 3. FT-IR spectra of caffeine, GO and rGO.
Thermal stabilities of graphite, GO and rGO were examined by TGA in a $\mathrm{N}_{2}$ atmosphere (figure 5). Graphite does not show any weight loss from room temperature to $800^{\circ} \mathrm{C}$. GO shows a $16 \%$ weight loss below $100^{\circ} \mathrm{C}$ as a result of the release of absorbed water, and a further rapid $24 \%$ weight
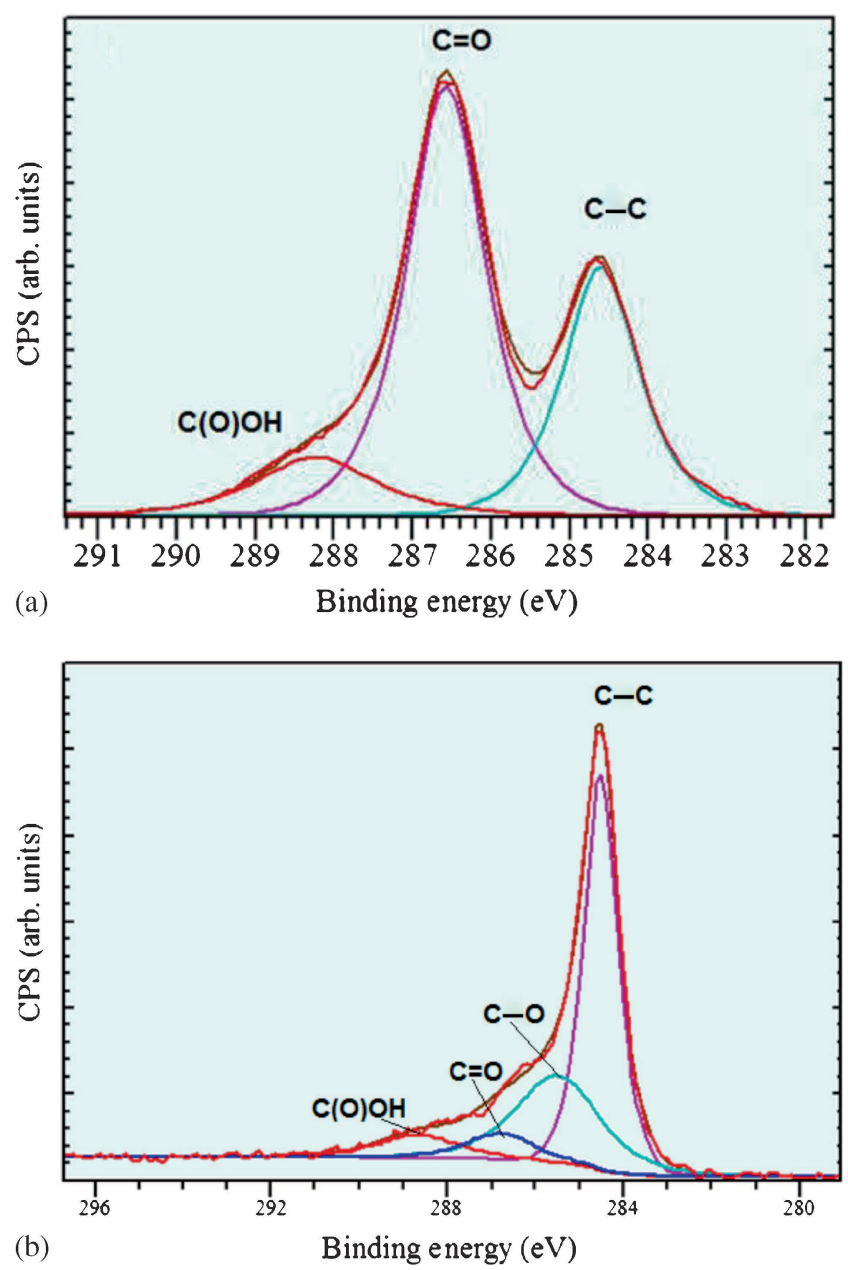

Figure 4. C 1s XPS spectra of (a) GO and (b) rGO.

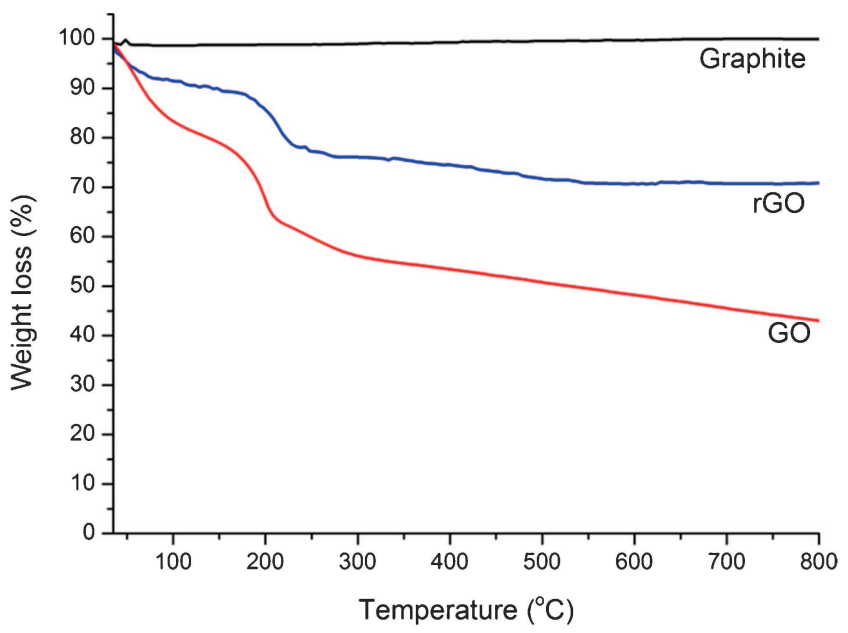

Figure 5. TGA plots of graphite, GO and rGO. 


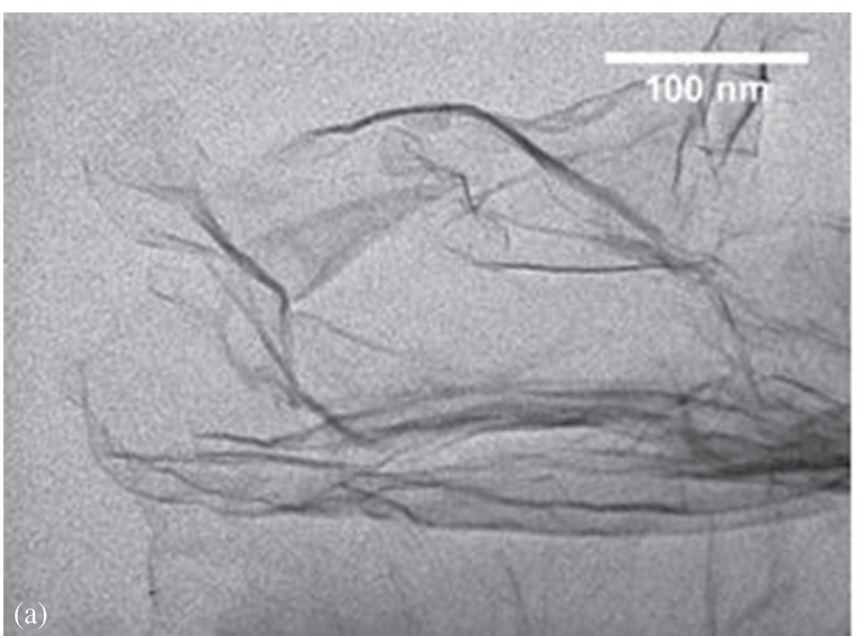

Figure 6. TEM images of (a) GO and (b) rGO.

loss from 100 to $250^{\circ} \mathrm{C}$ which is attributed to the removal of the oxygen-containing functional groups that yield $\mathrm{CO}$, $\mathrm{CO}_{2}$ or water. The weight loss of $\mathrm{GO}$ at $800^{\circ} \mathrm{C}$ is about $55 \%$. After reduction, the $\mathrm{rGO}$ exhibit a reduced total weight loss compared to GO ( $28 \%$ weight loss). These results indicate an obvious decrease in the amount of oxygen-containing functional groups and a profound reduction of GO by caffeine.

The morphologies of GO and as-prepared rGO were characterized using TEM. The TEM images of GO and rGO are shown in figure 6. A large GO sheets with dimension of micrometres have been observed (figure 6a), where it shows transparent and rippled silk wave appearances. From the TEM image shown in figure $6 \mathrm{~b}$, it can be seen that part of graphene sheet shows relatively flat morphology with low contrast, indicative of few-layer thickness.

\section{Conclusions}

In summary, rGO nanosheets were successfully produced by chemical reduction of GO using caffeine. They were characterized using various physical and chemical techniques. The use of caffeine as an alternative reductant to toxic chemicals provides a green and efficient method for the reduction of GO which makes it appropriate for large-scale production of graphene from natural graphite. In addition, after reduction process, caffeine and its derivatives can be easily removed from the product by washing. The obtained graphene nanosheets from natural raw graphite are potential biomaterial for many biomedical applications, including nanocarriers for targeting and localized drug delivery, bioimaging and biosensing.

\section{Acknowledgement}

We thank the Ministry of Science and Technology (Vietnam), for their support to this work through contract no. 101/2013/HD-NDT.

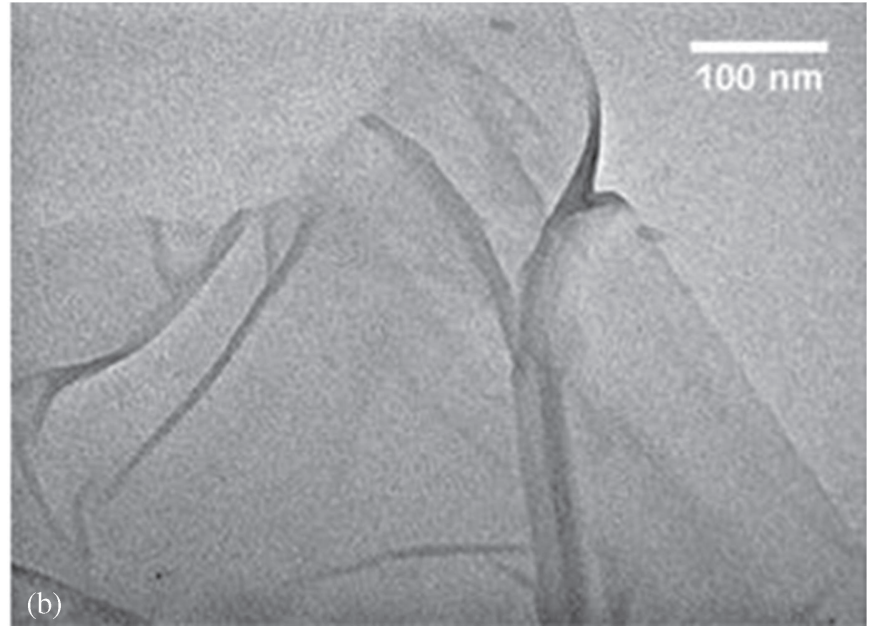

\section{References}

1. Haddon R C 2013 Acc. Chem. Res. 462191

2. Soldano C, Mahmood A and Dujardin E 2010 Carbon 482127

3. Dai L 2012 Acc. Chem. Res. 4631

4. Kim H, Kim S-W, Park Y-U, Gwon H, Seo D-H, Kim Y and Kang K 2010 Nano Res. 3813

5. Liu J, Cui L and Losic D 2013 Acta Biomater. 99243

6. Miao W, Shim G, Lee S, Lee S, Choe Y S and Oh Y-K 2013 Biomaterials 343402

7. Basu S and Bhattacharyya P 2012 Sens. Actuators B - Chem. 1731

8. Lee Y J, Park H W, Kim G-P, Yi J and Song I K 2013 Curr. Appl. Phys. 13945

9. Yan Z, Peng Z and Tour J M 2014 Acc. Chem. Res. 471327

10. Chia J S Y, Tan M T T, Khiew P S, Chin J K, Lee H, Bien D C S and Siong C W 2014 Chem. Eng. J. 249270

11. Xie G, Forslund M and Pan J 2014 ACS Appl. Mater. Interfaces 67444

12. Pei S and Cheng H-M 2012 Carbon 503210

13. Park S, An J, Potts J R, Velamakanni A, Murali S and Ruoff R S 2011 Carbon 493019

14. Akhavan O, Ghaderi E, Aghayee S, Fereydooni Y and Talebi A 2012 J. Mater. Chem. 2213773

15. Fernández-Merino M J, Guardia L, Paredes J I, Villar-Rodil S, Solís-Fernández P, Martínez-Alonso A and Tascón J M D 2010 J. Phys. Chem. C 1146426

16. Esfandiar A, Akhavan O and Irajizad A 2011 J. Mater. Chem. 2110907

17. Akhavan O, Kalaee M, Alavi Z S, Ghiasi S M A and Esfandiar A 2012 Carbon $\mathbf{5 0} 3015$

18. Gurunathan S, Han J W, Eppakayala V and Kim J-H 2013 Colloids Surf. B 102772

19. Ma Q, Song J, Jin C, Li Z, Liu J, Meng S, Zhao J and Guo Y 2013 Carbon 5436

20. Jana M, Saha S, Khanra P, Murmu N C, Srivastava S K, Kuila T and Lee J H 2014 Mater. Sci. Eng. B 18633

21. Pham T A, Kim J S, Kim J S and Jeong Y T 2011 Colloids Surf. A 384543 
22. Thakur S and Karak N 2012 Carbon 505331

23. Ferrari A C 2007 Solid State Commun. 14347

24. Tran D N H, Kabiri S and Losic D 2014 Carbon 76193

25. Park J S, Reina A, Saito R, Kong J, Dresselhaus G and Dresselhaus M S 2009 Carbon 471303
26. Garrigues J M, Bouhsain Z, Garrigues S and de la Guardia M 2000 Fresenius J. Anal. Chem. 366319

27. Stankovich S, Dikin D A, Piner R D, Kohlhaas K A, Kleinhammes A, Jia Y, Wu Y, Nguyen S T and Ruoff R S 2007 Carbon 451558 